\title{
Exploring the Value of Bedside Craniocerebral Ultrasound in the Dynamic Observation of Neonatal Subependymal Hemorrhage
}

\author{
Li Kaiwen \\ Department of Ultrasound, Jingzhou Central Hospital, The Second Clinical Medical College, Yangtze University, Jingzhou, China \\ Email address: \\ likaiwen1234@163.com

\section{To cite this article:} \\ Li Kaiwen. Exploring the Value of Bedside Craniocerebral Ultrasound in the Dynamic Observation of Neonatal Subependymal Hemorrhage. \\ International Journal of Biomedical Engineering and Clinical Science. Vol. 4, No. 1, 2018, pp. 1-5. doi: 10.11648/j.ijbecs.20180401.11
}

Received: January 3, 2018; Accepted: January 22, 2018; Published: February 7, 2018

\begin{abstract}
Background: The purpose of this study is to investigate the ultrasonic performance of bedside brain ultrasound in the observation of subependymal hemorrhages (SEH) in newborns and provide evidence for clinical prevention and treatment of hemorrhage. 152 cases of newborns diagnosed as SEH were examined and followed up regularly, and their ultrasonographic features were analyzed. As a result, the craniocerebral ultrasound found strong and slightly strong echo in the anterior horn of the lateral ventricle and the thalamic caudate nucleus region, which did not affect the lateral ventricle. In the early stage of hemorrhage, the lesions showed a high echo and the border was slightly blurred. The echo gradually increased and the boundaries became clear at the stable period. During the absorption period, the center of the lesion was low echogenic and the edge was still hyper echoic, and gradually formed a poorly sound, separated thick cyst cavity. Subsequently, there was no echo area in the center of the lesion, and eventually the lumen was formed under the subependymal hemorrhage. Post discharge follow-up showed that 6 cases (3.95\% 6/152), 75 cases (49.34\% 75/152), 37 cases (24.34\% 37/152), 13 cases (8.55\% 13/152) had their cyst cavity disappeared after their hemorrhage in 1 months and 2 months, 3 months and 6 months. The patients with their lesions basically absorbed within 6 months accounted for 86.18\% (131/152). Conclusion: Intracranial ultrasound has the advantage of early diagnosis of SEH, and it can be used to observe the patient's bedside examination, to observe the change of the course of the disease dynamically, to evaluate the blood transfer, to effectively guide the clinical medication and evaluate the prognosis.
\end{abstract}

Keywords: Bedside Craniocerebral Ultrasound, Neonate, Subependymal Hemorrhage, Dynamic

\section{Introduction}

Subependymal hemorrhage (SEH), also known as embryonic germinal matrix hemorrhage $(\mathrm{GMH})$, is a class I PVH-IVH (periventricle-intraventricle hemorrhage), which is a lighter type of PVH-IVH. If SEH is not discovered or treated timely, it may evolve into a severe type of PVH-IVH. $\mathrm{PVH}-\mathrm{IVH}$ is the intracranial hemorrhage most commonly seen in newborns, and is one of the main causes of early neonatal death. The bedside craniocerebral ultrasound is of great significance in the early diagnosis of neonatal SEH, dynamic observation of the evolution of the course of the disease, the assessment of the outcome of bleeding and the guidance of treatment. This study retrospectively analyzes the dynamic echocardiographic changes of 152 neonates with $\mathrm{SEH}$ who are treated and followed up in the hospital. It is reported as follows:

\section{Data and Methods}

\subsection{General Information}

From July 2014 to July 2017, 152 patients diagnosed as SEH by bedside sonography were enrolled in NICU of the hospital for various reasons, including 81 males and 71 females, gestational age at birth $(34.4 \pm 2.6)$ weeks. The causes of hospital admission included premature delivery (127 cases), low body weight (95 cases), pneumonia (24 cases), irritation (23 cases), scalp hematoma (19 cases), asphyxia (16 cases), hypoglycemia (15 cases), pneumothorax (15 cases), hyperbilirubinemia (13 cases), abnormal muscle tone (11 cases), vomiting (10 cases), poor feeding ( 8 cases) and diarrhea ( 7 cases). There were 127 preterm infants and 25 full 
term infants. One minute Apgar score was $8.24 \pm 1.25$, of which 7 cases were $\leq 3$; 98 were vaginal delivery ( 9 were obstetric forceps) and 54 were cesarean. On the day after hospitalization, the first bedside craniocerebral ultrasound examination should be performed, and the bleeding should be observed every 3 days. After discharge, the patients were asked to go back to the hospital at least once a month for reviewing the brain ultrasound until the lesions completely disappeared.

\subsection{Instruments and Methods}

When chosen Mindray M7 portable color Doppler ultrasound diagnostic apparatus, and the probe frequency of 8.0 MHz neonatal special probe for cranial ultrasound (C8-5). Make children supine and check them in sleep or quiet state. The examiner should be on the right or the head side of the children, the anterior fontanelle for coronal, sagittal section scan, the lateral fontanelle horizontal section scan, observe the standard cranial ultrasound section. Children who do brain ultrasound examination for first time should be at the bedside.

\subsection{SEH Diagnostic Criteria}

PVH-IVH is usually divided into the following 4 levels according to the Papile classification method, [1-2].

(1) level I: subependymal germinal matrix hemorrhage, coronal section in the anterior horn of lateral ventricle, the sagittal sulcus of caudate nucleus in the thalamus showing strong echo or a little strong echo, can occur in one side or both.

(2) level II: bleeding into the ventricles of the brain, but no ventricular dilatation.

(3) level III: intracerebroventricular hemorrhage with ventricular dilatation.

(4) level IV: intracerebroventricular hemorrhage accompanied by hemorrhagic infarction.

One level I PVH-IVH is SEH.

\section{Results}

\subsection{The Initial Ultrasound Diagnosis of SEH Children with Age and Number of Cases, the Percentage of the Situation in Table 1}

Table 1. The percentage of the initial ultrasound diagnosis of SEH children with age and number of cases.

\begin{tabular}{llllll}
\hline SHE newborns (days) & $\mathbf{1}$ & $\mathbf{2}$ & $\mathbf{3}$ & $\mathbf{4}$ & $\mathbf{1 5}$ \\
\hline Number of SHE cases (case) & $\mathbf{4 2}$ & $\mathbf{5 2}$ & $\mathbf{3 7}$ & $\mathbf{1 5}$ \\
\hline The percentage of total SEH & $27.63 \%$ & $34.21 \%$ & $24.34 \%$ & $9.87 \%$ & $2.63 \%$ \\
\hline
\end{tabular}

\subsection{SEH Ultrasound Performance}

Of 152 cases of SEH children, 50 cases of children with bilateral bleeding, 66 cases of children alone left hemorrhage, 36 cases of simple right haemorrhage. SEH brain coronal plane is found beneath the anterior horn of lateral ventricles with hyperechoic area, and parasagittal plane is found oval or irregular hyperechoic area in the thalamus, caudate sulcus, unswept lateral ventricle hemorrhage. The maximum diameter of the hemorrhage is $2 \sim 24 \mathrm{~mm}$. When the bleeding range is large, the oppression of the lateral ventricle caused by lesions makes the anterior horn vague.

\subsection{The Dynamic Changes of SHE}

In the early stage of hemorrhage, the lesions shows a high echo, and the border slightly blurs (Figure 1); in the steady-term, echo is slowly enhanced, boundaries clearing (Figure 2); in the absorption period, echo is low in the lesion center but still high in the edges, and gradually form a poorly sound, separated thick wall cavity; afterwards in the lesion center there appears echo-free zone, and finally become the form of subependymal hemorrhage cysts (Figure 3). 2 cases of SEH children who are after bleeding 3 to 5 days, hemorrhage breakthrough ependymal reach the lateral ventricle, and evolve into type III PVH-IVH, that is, intraventricular hemorrhage with ventricular dilatation (Figure 4).

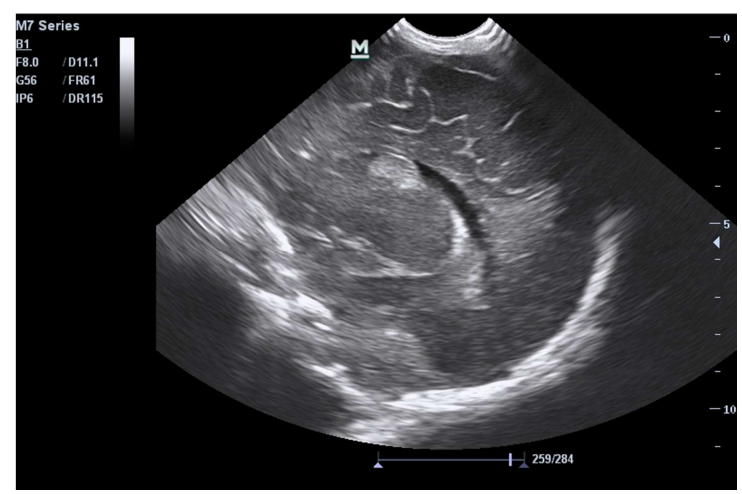

Figure 1. Hyperechoic in the lateral anterior horn of the left ventricle and the thalamus caudate nucleus.

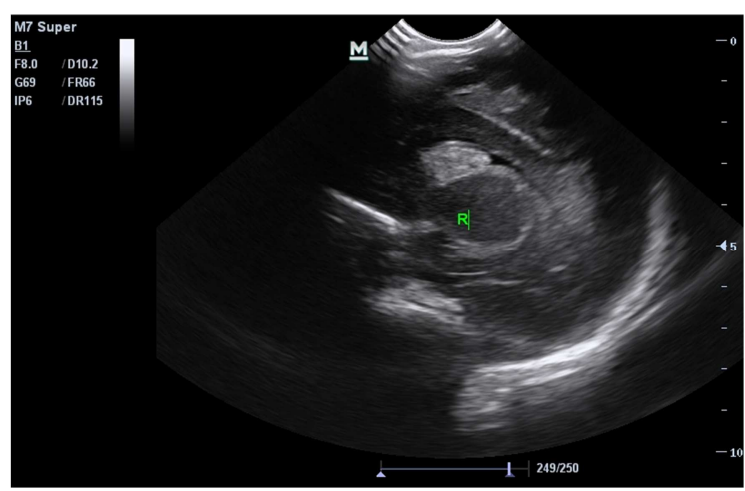

Figure 2. Strong echo group of the right ventricle of the right ventricle and the hypothalamic caudate nucleus. 


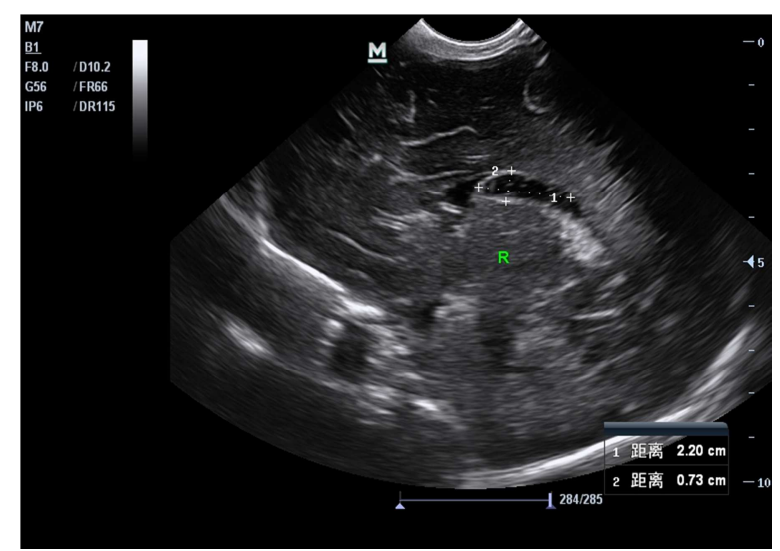

Figure 3. Anechoic cystic cavity of the anterior right ventricle of the right ventricle and the thalamus caudate nucleus.

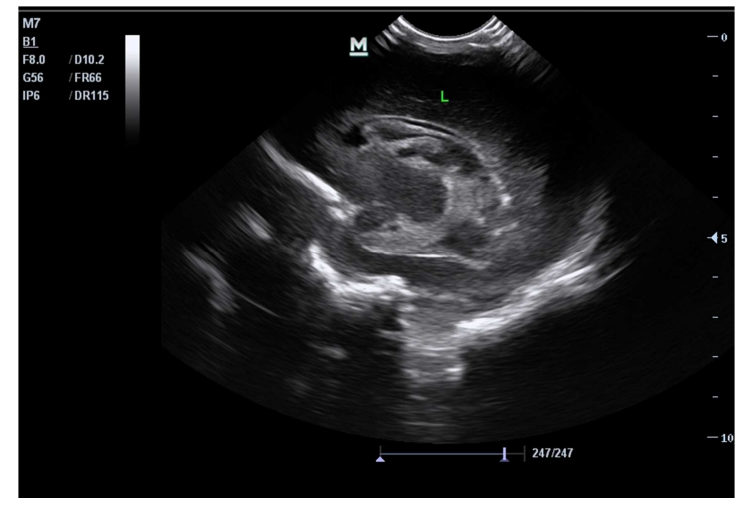

Figure 4. Left ventricular hemorrhage with ventricular enlargement.

\subsection{SEH Follow-up}

Of 152 followed-up patients with SEH, 6 cases $(6 / 152$, $3.95 \%$ ) are that cysts disappeared in 1 months after subependymal hemorrhage; 75 cases $(75 / 152,49.34 \%)$ are that when children are 2 months, cysts disappear after hemorrhage; 37 cases $(37 / 152,24.34 \%)$ are that when children are 3 months, the cystic cavity disappear; 13 cases $(13 / 152,8.55 \%)$ are that when children are 6 months, the cystic cavity disappear; 11 cases $(11 / 152,7.24 \%)$ are that when children are 8 months, the cystic cavity disappear. And 18 of the children are accompanied by mild ventricular widening.

\section{Discussion}

$\mathrm{SEH}$ is common in preterm infants, and the smaller the gestational age is, the higher the incidence of SHE is, which may be related to the brain maturation and hemodynamics of the newborn. Antoniuk et al [3] reported that grade I hemorrhage in subependymal germinal vesicle intraventricular hemorrhage in preterm infants with gestational age less than 32 weeks and / or birth weight less than $1500 \mathrm{~g}$ accounts for $70 \%$. Of 152 cases of SEH in this group of children, preterm children are up to $83.55 \%$ $(127 / 152)$. The site of SEH hemorrhage is mainly under the ependymal stroma, that is, embryonic germinal layer matrix where the caudate nucleus and thalamic junction meet.
Embryonic germinal layer matrix is a kind of immature juvenile capillaries, composed of primitive neurons and glial cells of nerve tissue, which form the core structure of the cerebral cortex and the deep. The matrix consists of thin-walled capillaries containing only monolayer of endothelial cells, lacking connective tissue support, fragile and easily damaged. At 28 32 weeks of gestational age, the germinal matrix is active to divide and is rich in mitochondria. It is easy to undergo necrosis and disintegration and lead to $\mathrm{SEH}$ in hypoxia, acidosis and hypercapnia. With the increase of fetal age, the volume of the stroma in the embryonic germinal layer decreases, atrophies, and basically degenerates before 36 weeks. Therefore, special attention should be paid to the screening of craniocerebral ultrasound for premature infants, so as to make early diagnosis and intervention. The blood supply to the embryonic germinal layer matrices is mainly provided by capillary networks consisting of the anterior cerebral arteries, the striatum of the middle cerebral artery, and the distal end of the internal carotid artery, and finally to the deep venous system, which is specifically U-shaped. When subjected to risk factors (such as vasodilation caused by cerebral ischemia or hypoxia), it may cause changes in cerebral blood flow and systemic blood pressure fluctuations, which can cause the capillary stripping of the SEH. Therefore, perinatal asphyxia, vaginal delivery, forceps midwifery, inappropriate mechanical ventilation can induce SEH.

The bleeding sites of SEH can be unilateral or bilateral. In this group, left side $>$ bilateral side $>$ right side and SEH on the left side are common in this group. The specific reasons need to be analyzed. The basic sonogram of SEH shows a high echo in the lateral inferior ventricle and the caudate sulcus of the thalamus. It is due to the formation of the interface caused by the acoustic impedance of blood, which is higher than that of the cerebral parenchyma and cerebrospinal fluid. In the initial stage of blood splatter, the lesion forms hyperechoic mass due to cellulose deposition and clot formation. At this time, the patient should be kept quiet to avoid moving or irritating operation as much as possible. Vitamin K1, hemostasis Min, etc., should be prevented for further development of bleeding. At this stage, the size of the hemorrhage is different, the boundary is regular or irregular, which is related to the amount of bleeding or the speed of bleeding. After 2 3 days of bleeding, if the focus of the lesion is stronger and the boundary is clearer, it shows that the blood clot is contracted and has entered a stable period. When re-examined children in the brain cranial ultrasound after 5 to 7 days' bleeding, if the situation is stable, then discharge and ask them to return to hospital on schedule; but if the bleeding area is larger, the lesion oppresses anterior chamber angle so much so that it gets deformation and breaks into the lateral ventricle, it will cause varying degrees, asymmetric ventricular enlargement, and even break through the ventricular wall to invade the brain parenchyma. Severe intraventricular hemorrhage can be obstructed and adhered by bleeding, causing obstructive hydrocephalus by poor cerebrospinal fluid circulation In addition subependymal 
germinal layer matrix hemorrhage can block the terminal vein, affecting the local myelodystrophy and hemorrhagic infarction, and even the formation of hole brain and the poor prognosis. The children of SEH in this group are rechecked regularly after clinical treatment. The dynamic observation of craniocerebral ultrasound shows that the process needs 2 3 weeks, which is basically anastomosed with the related research [4-5]. Hospital SEH children should go back for at least a one-time review of cranial ultrasound after discharge in order to understand whether cysts disappear, ventricle expanse and so on, and according to the size of children' head circumference, fontanelle tension, muscle tension, the original reflex, adjust their number of ultrasound review; At the same time, the neurodevelopment of the children is evaluated by the combination of behavioral neuromeasurement and electroencephalogram. In this group 152 cases are followed up, 6 cases $(6 / 152,3.95 \%), 75$ cases $(75 / 152,49.34 \%), 37$ cases (37 / 152, 24.34\%) show that children' cysts disappear in 1, 2, 3 months after hemorrhage, that is within 3 months, the recovered children account for $77.63 \%$ (118 / 152).

SEH belongs to the mild PVH-IVH. Hospitalized causes of 152 cases include premature birth, low birth weight, pneumonia, irritability, scalp hematoma, pneumothorax, asphyxia, hypoglycemia, hyperbilirubinemia, dystonia, vomiting, diarrhea, and poor feeding. Mostly those who have no or only mild neurological symptoms and signs are found $\mathrm{SEH}$ in the brain ultrasonic inspection. In the past, patients with mild PVH-IVH had a good prognosis, so most of them were not given enough attention. But now research [6] shows that even children with mild PVH-IVH who lack typical clinical symptoms may have poor prognosis in school age and adolescence, such as motor and mental retardation, epilepsy, cerebral palsy and so on. Early diagnosis and effective symptomatic treatment of children with SEH are beneficial to reduce neurological impairment and disability rate for their neonatal brain plasticity and reproductive ability. The group of 152 children at the birth of 1 day, 2 days, 3 days who are detected SEH are 42 cases, 52 cases and 37 cases, that is, three days after birth, SEH is detected in 131 cases, accounting for $86.18 \%(131 / 152)$ Maybe it is associated with significantly lower platelet activity in neonates during 3 to 4 days of birth than adults. It was suggested that it should be better to complete the first cranial ultrasound examination within 3 to 4 days after birth in order to detect and treat early SEH children with no obvious clinical symptoms. Currently, of the most common used medical imaging methods, CT has radiation and MRI is expensive and less sensitive to fresh bleeding. Besides most of them need to move the child, easily leading to fluctuations in blood pressure and increasing the risk of intracranial hemorrhage. And craniocerebral ultrasound examination is cheap, noninvasive, and bedside operation. It is especially suitable for severe children who are not fit to move, so it is convenient for dynamic observation. The range of SEH is generally small. The minimum diameter in this group is only $2 \mathrm{~mm}$, and CT and MRI are not easy to detect it. Research [7-9] shows that ultrasound has high specificity and sensitivity for the central lesion of brain. It has more advantages in diagnosing SEH than CT and MRI. Early detection of small SEH hemorrhage can be found. Ultrasound can also conjecture the bleeding phase of SEH [10], and make a clear judgement of the degree of bleeding to guide clinical targeted treatment. Because ultrasound is very sensitive to very low concentration of hemoglobin, the diagnosis of intracranial hemorrhage can be as late as 3 months or more. Craniocerebral ultrasound screening is the main means of early diagnosis, tracking observation and follow-up evaluation of SEH.

\section{Conclusion}

In summary, using bedside cranial ultrasound can early detect and have a dynamic observation of neonatal SEH: This study shows that within 1 weeks SEH lesions mainly appear high and strong echo; then from the central site, lesions begin to liquefy and absorb, and gradually form after-hemorrhage sac, this process takes about 2 to 3 weeks; more than $75 \%$ cysts can disappear in 3 months. The bedside craniocerebral ultrasound is of great significance in the early diagnosis of $\mathrm{SEH}$, the dynamic observation of the course of the disease, the assessment of the outcome of bleeding and the guidance of clinical treatment.

\section{References}

[1] Neonatology Group of Chinese Medical Association Pediatric Branch, Editorial board of the Chinese Journal of Pediatrics. Diagnosis of periventricular- intraventricular hemorrhage and periventricular leukomalacia in premature infants [J]. Chinese Journal of Pediatrics, 2007, 45 (1): 34-36.

[2] Papile LA, Burstein J, Burstein R, et al. Incidence and evolution of subependymal and intraventricular hemonhage: a study of infants with birth weights less than 1,500gm [J]. J Pediatr, 1978, 92 (4):529-534.

[3] Antoniuk S, da Silva RV. Periventricular and intraventricular hemorrhage in the premature infants [J]. Rev Neurol, 2000, 31 (3):238-243.

[4] Lu Jun, Li Jiangling, Liu Yingqiong. Transcranial Ultrasound Diagnosis of 92 Cases of Neonatal Intracranial Hemorrhage [J]. China Practical Medicine, 2010, 5 (29):41-43.

[5] Lin Shulian, Bu Qiuqiang, Xian Yunkai. The Value of Craniocerebral Ultrasound in Neonatal Brain Disease [J]. China Practical Medicine, 2012, 7 (27):98-99.

[6] Van den Broeck C, Himpens E, Vanhaesebrouck P, et al. Influence of gestational age on the type of brain injury and neuromotor outcome in high-risk neonates [J]. Eur J Pediatr, 2008, 167 (9):1005-1009.

[7] Zhou Congle. Neonatal Neurology [M]. Beijing: People's Medical Publishing House, 2012, 321-329.

[8] Pei Yufang, Zhang Lianjun, Zhang Hong. Study and Analysis of Diagnosis of Intracranial Hemorrhage in Newborns by Craniocerebral Ultrasound and CT $[\mathrm{J}]$. Chinese Journal of Preventive and Genetic, 2015, 23 (1): 83-84. 
[9] Zhao Yali, Dong Jiao Lou, Zhou Cexun. The clinical value of color Doppler ultrasound and CT examination of the brain in newborns [J]. Medical Imaging Journal, 2013, 23 (6): 969-970.
[10]Liu Jiaoran, Li Tao, Liu Fang. Study on early neonatal ependymal hemorrhage under hemodynamics [J]. Journal of clinical ultrasonic medicine, 2011, 13 (4):238-240. 